\title{
Predicados proyectables y adquisición de palabras
}

Jahaziel Estefanía Cervantes López Universidad Autónoma del Estado de Morelos lopez.filos@gmail.com

\section{Introducción}

La presente investigación pretende ser un puente entre algunas teorías que encontramos en la filosofía y los trabajos experimentales de las ciencias cognitivas, específicamente, de la adquisición de palabras, como los trabajos de Dedre Gentner y Ellen Markman.

La idea de reunir el trabajo teórico de la filosofía y el trabajo empírico de la adquisición de palabras cumple el objetivo de enriquecer $\mathrm{o}$, si se quiere, naturalizar los aportes del filósofo Nelson Goodman, así como el de estimular el trabajo transdisciplinar.

Lo que refleja esta tesis es la plausibilidad de caracterizar el aporte teórico de Nelson Goodman a la discusión de la inducción: los predicados proyectables, por medio de contribuciones encontradas en los resultados y teorías de la ad- 
quisición de palabras ${ }^{1}$, concretamente, como lo hemos mencionado, con los aportes de Dedre Gentner y Ellen Markman.

Así pues, el objetivo principal de este trabajo es caracterizar los predicados proyectables, es decir, determinar los rasgos que hacen distinto a un predicado usado en nuestras inducciones -en este caso en una forma de inducción conocida como proyección-, de otro que no se puede usar en nuestras proyecciones, llamado predicado no proyectable por Goodman. Para lograr esto, nos centramos en un caso de estudio particular tomado del mismo Nelson Goodman: el predicado verde -como predicado proyectable- y el predicado verzul -como predicado no proyectable-. La manera que proponemos para caracterizar verde y verzul es por medio de un espacio abstracto descriptivo, como los usados en matemáticas y ciencias naturales.

Con el análisis de verde y verzul como nuestro caso de estudio, pretendemos que, al caracterizarlos, esto nos dé la pauta para futuras investigaciones que girarán en torno a la proyectabilidad. Es decir, pretendemos que, al tomar como punto de referencia los resultados arrojados de este caso de estudio, esto nos permita, eventualmente, generalizar nuestro aporte, poder separar «la región proyectable» de «la región no proyectable» en el espacio descriptivo.

Antecedentes. Inducción: Nelson Goodman

En 1695, Sir Edmon Halley estaba calculando las órbitas de un conjunto de cometas para su inclusión en los Principia Mathematica de Newton cuando notó una sorpresiva regularidad: los cometas de 1531, 1607 y 1682 
tomaron caminos notablemente similares en el cielo, y visitaron la Tierra con aproximadamente 76 años de diferencia. Newton ya había mostrado que los cometas deberían seguir órbitas correspondientes a secciones cónicas -parábolas, hipérbolas y elipsis- aunque ninguna órbita elíptica había sido observada. Halley infirió que los avistamientos de estos cometas no eran tres eventos independientes, sino tres consecuencias de una causa común: un cometa que había visitado la Tierra tres veces y viajaba en una órbita elíptica. Él llegó a predecir que éste regresaría a lo largo de la misma órbita en 1758. El cometa volvió como lo había predicho, y ha seguido visitando la Tierra aproximadamente cada 76 años desde entonces, proporcionando una confirmación sensacional a la física de Newton (Griffiths, 2009: 661).

El razonamiento inductivo ha ocupado un gran espacio en las discusiones filosóficas de todos los tiempos debido a su uso y utilidad en la vida diaria y en la práctica científica. Para abordar el tema de la inducción es importante sintetizar qué es lo que se entiende cuando hablamos de ella y posteriormente poder hilarla al tema que nos compete: los predicados proyectables.

Con la cita anterior de Griffiths pretendemos mostrar un caso en ciencia en el cual se ilustra claramente cómo se comporta la inducción. La inducción está sustentada en las observaciones que se hacen de determinado fenómeno. La observación de distintos eventos o fenómenos -la observación de cometas en 1531, 1607 y 1682- nos permite hacer inferencias sobre fenómenos particulares como, en el ejemplo, inferir una causa común para los avistamientos de cada uno 
de los cometas: que las observaciones en diferentes años nos indican que puede tratarse de un mismo cometa y que ese cometa en particular, entonces, viaja en órbita elíptica.

Además de este tipo de inferencias que resultan de la inducción -el reconocimiento de una causa común-, empleamos la inducción para producir generalizaciones del tipo «cada 76 años el cometa Halley visita la Tierra», tomando las regularidades observadas en los fenómenos -es decir, que en 1531 el cometa $x$ ha visitado la Tierra, que en 1607 el cometa $x$ ha visitado la Tierra, etcétera-. También la inducción nos permite hacer predicciones tales como «el cometa Halley visitará la Tierra dentro de 76 años más a partir del momento t».

Nótese que el rasgo fundamental que hace distinto y controversial al tema de la inducción es que las hipótesis arrojadas por medio de este razonamiento están apoyadas en cuestiones empíricas, lo que lo hace diferente del tipo de razonamiento erigido desde los griegos: el razonamiento deductivo. Para establecer la diferencia manifiesta entre ambos tipos de razonamientos, basta decir que el razonamiento inductivo se inicia con observaciones particulares que nos llevan, después de determinado número de observaciones, a una generalización o a una proyección como las antes mencionadas. Para este tipo de razonamiento no existen herramientas lógicas ${ }^{2}$ que nos permitan establecer la confirmación de la conclusión, o dicho de otro modo, de la hipótesis que se genera. Contrario al razonamiento inductivo, el razonamiento deductivo principia con una hipótesis o ley establecida y, a través de diversas inferencias que se posibilitan gracias a he-

2 Nos referimos a las reglas de introducción y reglas de eliminación de cada uno de los conectivos existentes en la lógica de primer orden, a saber, la conjunción, disyunción, condicional, etc. 
rramientas lógicas, se demuestra o no la verdad de nuestras conclusiones. En resumen, la inducción se trata de confirmación y la deducción se trata de demostración.

Volviendo al ejemplo dado por Griffiths, y teniendo presente la caracterización que hemos hecho del razonamiento inductivo, podemos preguntar: ¿qué es lo que le aseguró a Halley que el cometa que había visitado tres veces la Tierra lo volvería a hacer?, ¿hay alguna manera de justificar su predicción? Este tipo de preguntas ilustran lo que es conocido como el problema de la justificación de la inducción.

Si bien este modo de razonar -es decir, inductivamentepuede resultarnos muy familiar dado que la ciencia moderna procede de este modo, es decir, haciendo observaciones de los fenómenos y reportando su regularidad, según David Hume, filósofo empirista del siglo XVIII, no existe justificación para este modo de proceder. Si Hume hubiera dado una respuesta al caso particular del cometa, diría que lo único que justificó a Halley para predecir que ese cometa volvería a visitar la Tierra fue la regularidad que vio en el pasado, cerrando con ello la oportunidad de una posible justificación al problema de la inducción.

Sin embargo, en la filosofía contemporánea, el problema de la justificación de la inducción ha sido reformulado en el Nuevo enigma de la inducción de Nelson Goodman. Para Goodman el problema debe de ser reinterpretado como un problema de descripción y explicación, y no de justificación, que tendría que responder a preguntas tales como ¿cuál es la diferencia entre una inducción válida y una inducción no válida? (Rheinwald, 1993).

Así, justo en esta parte de la historia es preciso detenerse, ya que es del trabajo de Goodman del que surge el problema de la presente investigación. Por ello, a continuación, pre- 
sentamos una reseña de El nuevo enigma de la inducción, para mostrar el marco general bajo el cual se desarrollan sus ideas y que podamos entender de dónde emergen los predicados proyectables.

Nelson Goodman y el nuevo enigma de la inducción

Retomando la pregunta que enunciamos en el apartado anterior sobre la diferencia entre una inducción válida y una inducción no válida, mostramos apenas una de las incógnitas que Goodman formula cuando habla del nuevo enigma de la inducción y una de las más importantes para nuestra investigación. Los esfuerzos de Goodman no están enfocados a describir el razonamiento inductivo, sino que esa respuesta, según él, ya la había dado Hume al decir que la mente es llevada por un impulso a hacer la transición de regularidades vistas en el pasado, hacia eventos no observados: eventos futuros (Goodman, 1953). Sin embargo, Goodman quiere saber qué hace lícita la elección de unas proyecciones -afirmaciones sobre el futuro- más bien que de otras. Y es esto el llamado nuevo enigma de la inducción, al cual también se le conoce como el problema de la confirmación ${ }^{3}$.

El tema de la confirmación está estrechamente relacionado con el tema de la inducción y es tan controversial como éste. Sea suficiente con que rescatemos que la confirmación es la relación que existe entre la evidencia y cualquier oración que sea soportada por esa evidencia. La confirmación puede adquirir dos sentidos: 1) el sentido incremental, en el cual una pieza de evidencia contribuye, al menos en algún

3 No olvidemos que Goodman se refiere a confirmación haciéndola equivalente a inducción válida, proyección válida e hipótesis legaliforme. 
grado, a dar soporte a la hipótesis en cuestión, y 2) el sentido absoluto, «en el cual un cuerpo de evidencia provee un fuerte soporte para la hipótesis en cuestión»(Audi, 1999: 172). Cabe mencionar que, dentro de las paradojas de la confirmación, se encuentra la muy conocida Paradoja de los cuervos formulada por Carl Hempel y la Paradoja del verzul, introducida por Goodman.

Entrados pues en el caso de la confirmación, Goodman propone distinguir hipótesis como «todo el cobre conduce electricidad» de otras como «todos los hombres en este cuarto son el tercer hijo». Las primeras son llamadas «legaliformes»y las segundas «accidentales». En el caso de las primeras, con la aparición de diferentes instancias, la credibilidad de la oración aumenta y confirma la hipótesis en cuestión, a saber, que todo el cobre conduce electricidad. No obstante, con las segundas esto no pasa, es decir, no se confirman con algunas instancias observadas. La cuestión entonces es, ¿cómo distinguimos hipótesis legaliformes de hipótesis accidentales?

Para indagar sobre dicha cuestión, Goodman propone un ejemplo con esmeraldas. Por un lado, tenemos una hipótesis del tipo «todas las esmeraldas son verdes» $\mathrm{y}$, por el otro, una que afirma «todas las esmeraldas son verzules». En el caso de la primera afirmación, tenemos una serie de observaciones ${ }^{4}$ que, hasta este momento, digamos $t$, soportan la hipótesis de que todas las esmeraldas son verdes. En el segundo caso tenemos una afirmación con un predicado menos familiar, «verzul», que debe ser definido. «Verzul» es un predicado acuñado por Goodman y se refiere a «todas las cosas examinadas antes de $t$ sólo en caso de que sean verdes, pero a otras cosas sólo en caso de que sean azules»

$4 \quad$ Esto es: $a$ es una esmeralda y $a$ es verde, $b$ es una esmeralda y $b$ es verde, y así. 
(Goodman, 1953: 74). Antes del tiempo $t$ tenemos una serie de observaciones que afirman que «todas las esmeraldas son verdes» $\mathrm{y}$ «todas las esmeraldas son verzules». Las mismas observaciones confirman ambas hipótesis ${ }^{5}$ hasta el tiempo $t$; sin embargo, en el caso de verzul sabemos que si no es observado antes de $t$ nuestro predicado tiene que aplicarse a esmeraldas azules. Y dado que ambas hipótesis se confirman, Goodman afirma que, sin embargo, «estamos conscientes de cuál de las dos predicciones incompatibles está genuinamente confirmada» (Goodman, 1953: 74).

Como resultado de este razonamiento, Goodman concluye que, eligiendo el predicado apropiado, obtendremos la confirmación de nuestra hipótesis. La pregunta ahora es, ¿cómo determinamos que un predicado sea apropiado para confirmar una hipótesis? Es decir, ¿cómo saber que verde -en el caso de las esmeraldas- confirma mejor nuestra hipótesis que verzul? La respuesta de Goodman es: gracias al atrincheramiento. El atrincheramiento depende de la frecuencia de proyección y nosotros, dice Goodman, podemos hacer esta distinción -de que verde está mejor atrincherado que verzul- gracias al historial de proyecciones pasadas que tiene la clase en la que está incluido el predicado verde; en otras palabras, la clase a la que pertenece verde está mejor atrincherada debido a la gran cantidad de veces que verde, $u$ otro de los miembros de esa clase, se usó y proyectó en diferentes hipótesis, comparado al predicado verzul.

Ya que hemos reseñado de manera suficiente El nuevo enigma de la inducción, hay que señalar que de este aporte de Goodman -del atrincheramiento y del uso para justificar la elección de un predicado más bien que de otro- es precisa- 
mente de donde surge nuestro problema de investigación. Consideramos que el uso y el atrincheramiento de las palabras es una idea incompleta que puede ser robustecida con los aportes en áreas de investigación de las ciencias cognitivas, específicamente con resultados de investigaciones en adquisición de palabras.

La idea de que el resultado de Goodman es incompleto no debe ser mal entendida: lo único que pretendemos al robustecer su aporte es mostrar una alternativa a su explicación. Consideramos que su idea es incompleta debido a que, en el tema de la inducción, Goodman está teniendo en cuenta un aspecto sumamente importante además de la mera forma lógica, es decir, el contenido semántico de la inducción. Si Goodman está proponiendo enfocarnos en el contenido de las inducciones, en las palabras que son usadas, deberíamos de considerar a las palabras mismas para explicar su posible proyección -en este caso enfocándonos en la adquisición de estas palabras-, y no únicamente en el promedio de uso de éstas.

\section{Planteamiento del problema}

Dicho lo anterior, basta ahora concentrarnos en los predicados proyectables. Como ya se dijo, al hablar de predicados proyectables, Goodman está centrando su atención en el contenido del razonamiento inductivo, es decir, no se enfoca en el cálculo que pudiera hacerse si tuviéramos las herramientas lógicas necesarias para obtener hipótesis verdaderas -como sucedería con el razonamiento deductivo, que hemos caracterizado en el apartado de antecedentes- sino al contenido 
mismo, si se quiere, al significado de las palabras implicadas en el razonamiento inductivo.

Sin embargo, y dado que consideramos incompleta la respuesta del atrincheramiento de Goodman, creemos que su aporte: 1) no nos permite distinguir claramente un predicado proyectable de un predicado no proyectable y 2) estamos convencidos de que está implicado algo más en el hecho de que un predicado sea proyectable que el mero uso o atrincheramiento de las palabras.

Como resultado de lo anterior, nos preguntamos: ¿podrían las ciencias cognitivas ofrecer una caracterización que nos permita separar predicados proyectables de predicados no proyectables? Si esto es así, ¿cómo es que pueden lograrlo?

Sostenemos que la caracterización de un predicado proyectable gracias a las ciencias cognitivas es posible, más concretamente, que las teorías y resultados en adquisición de palabras (en desarrollo del lenguaje), con investigadoras como Dedre Gentner y Ellen Markman, permiten identificar rasgos característicos ${ }^{6}$ de los predicados proyectables y no proyectables. Estos rasgos característicos que es posible identificar con las investigaciones en ciencias cognitivas tácitamente, en investigaciones de desarrollo del lenguajecumplen lo siguiente:

1. son valores en la información relevantes en la constitución de conceptos;

2. la variación de esos rasgos o regiones que es posible identificar en estas investigaciones, constituye un continuo;

6 Donde con rasgos queremos decir «unidades psicológicas de percepción o pensamiento» (Cohen 2005; 672). 
3. estos continuos podemos interpretarlos como ejes o dimensiones que nos permiten construir un espacio descriptivo y, por lo tanto,

4. este espacio nos permite caracterizar y diferenciar los predicados proyectables y no proyectables.

Así pues, ya que hemos planteado nuestro problema y propuesto una aproximación para su solución, exponemos a continuación una sinopsis de lo que vendrá en capítulos posteriores.

Sumario

Plantearemos las inquietudes sobre la propuesta de Goodman y esbozaremos las razones por las que las investigaciones en adquisición de palabras son útiles para caracterizar un predicado proyectable; además, esbozaremos las ideas relevantes de Dedre Gentner y Ellen Markman para mostrar cómo pueden ayudar en dicha tarea.

Después, expondremos nuestra propuesta: integraremos los elementos que hemos expuesto anteriormente para mostrar cómo pretendemos que den solución a nuestro problema de caracterizar los predicados proyectables y no proyectables. También clarificaremos los aspectos fundamentales de dicha solución y las condiciones bajos las cuales ésta puede llevarse a cabo desde nuestra perspectiva.

Posteriormente expondremos cómo es que funciona dicha solución haciendo uso de los trabajos de Gentner y Markman, lo cual nos permitirá llegar a la separación de predicado proyectable y predicado no proyectable.

Finalmente, expondremos las conclusiones y nuevas perspectivas para robustecer, en un futuro inmediato, la so- 
lución a la separación de un predicado proyectable y de un predicado no proyectable.

Adquisición de palabras

La solución de Goodman para distinguir un predicado proyectable como verde, de un predicado no proyectable como verzul, radica en el atrincheramiento de las palabras. Como ya se ha mencionado, este atrincheramiento deriva del uso y es lo que hace que verde sea una palabra más primitiva comparada con verzul. Ésta es la razón dada por Goodman sobre nuestra preferencia de una palabra sobre otra. Nuestra inconformidad radica, entonces, en que sea el mero uso y el atrincheramiento el que determine nuestra preferencia al proyectar unas palabras más bien que otras.

Para completar la idea de Goodman, en aras de mantener la idea de que existen cosas tales como los predicados proyectables en una inducción exitosa, nos preguntamos lo siguiente: ¿existe algo más que el uso o atrincheramiento de las palabras que permita justificar nuestra preferencia por unas palabras más que por otras? Como, por ejemplo, pensando en nuestro estudio de caso que se trata de términos de color, la saliencia del color amarillo vs el color café o «el orden sistemático de adquisición» (Pitchford, 2002: 1350) de las palabras que hace que un niño de 4 años de edad prefiera (produzca y comprenda) un término de color en lugar de otro. En afinidad con este tipo de evidencia, proponemos que los resultados de las investigaciones de adquisición de palabras pueden usarse para describir lo que Goodman llama predicados proyectables. 
Para indagar sobre dicha cuestión, utilizaremos los trabajos de Gentner y Markman, quienes han trabajado en la adquisición de palabras como sustantivos, adjetivos y verbos. Esperamos que nos permitan desarrollar una solución al problema de caracterizar un predicado proyectable. Así, en los siguientes tres apartados, sintetizaremos las ideas más relevantes de estas investigaciones.

\section{Dedre Gentner: el aprendizaje temprano de palabras}

Gentner (1982), propone que los sustantivos son conceptualmente más básicos que los conceptos referidos por los verbos o preposiciones. Esta distinción está basada en aquella que diferenciaba a «la hipótesis de la partición natural» de «la relatividad lingüística». La hipótesis de la partición natural sostiene que «hay en el flujo experiencial ciertas colecciones de preceptos que son universalmente conceptualizados como objetos»(Gentner, 2001: 217) -a saber sustantivos y predicados-. Mientras que la relatividad lingüística sostiene que «esta aparente diferencia conceptual»-entre sustantivos y predicados, por un lado, y verbos y preposiciones, por el otro- «sea realmente una ilusión impuesta sobre nosotros por nuestro lenguaje. [...] es el lenguaje el que establece las distinciones entre las partes del habla» (Gentner, 1982: 302).

De estas divisiones se desprenden, según Gentner y de acuerdo con nociones estándar en lingüística, la «clase abierta» y la «clase cerrada». La primera es una «gran categoría léxica que fácilmente acepta nuevos miembros - e.g. sustantivos, verbos y adjetivos- y la clase cerrada es una típicamente pequeña categoría léxica en la que nuevos miembros son raramente agregados -por ejemplo, preposiciones, determinantes y conjunciones» (Gentner, 2001: 216). 
Con lo anterior, Gentner justifica su propuesta, que presentamos a continuación: existe un continuo que tiene en los extremos, por un lado, el «dominio cognitivo» y, por el otro, el «dominio lingüístico». Esta división se desprende de las dos divisiones anteriores, sólo que para Gentner no es preciso verlas como disyuntivas, sino que, más bien, hay que considerarlos aplicándose en diferentes grados y diferentes tipos de palabras. Es decir, es posible ver el desarrollo conceptual del niño comenzando por un extremo del continuo: el dominio cognitivo. En el dominio cognitivo «los conceptos surgen de una esfera cognitivo-perceptual y son simplemente nombrados por el lenguaje» (Gentner, 2001: 215); en este extremo se identifican los nombres propios y sustantivos. A lo largo de este desarrollo, se transita por términos de relación entre objetos, verbos, preposiciones espaciales, hasta llegar al otro lado del extremo con los determinantes y conjunciones, que pertenecen al dominio lingüístico. En este dominio «el mundo presenta un pedazo perceptual cuyo aglutinamiento no está pre-ordenado, y el lenguaje dice cómo los pedazos quedan combinados en conceptos»(Gentner, 2001: 215).

Ellen Markman: Adquisición de términos de categoría

En este capítulo del libro titulado Categorización y nombramiento en niños, problemas de inducción, Markman propone una teoría de cómo es que los niños categorizan el mundo. Concretamente, si se basan en «relaciones temáticas» o en «relaciones taxonómicas». Una relación temática se refiere a las relaciones entre objetos que no son capturadas por el análisis interno de sus propiedades; éstas pueden ilustrarse con las siguientes oraciones: «estar al lado de...», «estar encima de...», «los humanos leen libros», «los perros mastican huesos», etc. (1989: 21). Mientras que una categorización ta- 
xonómica es más bien «la existencia de ciertas características (perceptuales, funcionales o algo más abstracto), que son compartidas por los ejemplares de una categoría y que los distingue de los ejemplares de otra categoría» (1989; 21). Lo que distingue a estas dos clasificaciones es que la primera se refiere a la participación de dos objetos en un evento o tema en particular, mientras que la segunda se refiere a los rasgos o características que comparten de manera necesaria o probabilística los objetos.

Aludiendo al dilema de Quine -conocido también como el problema de la subdeterminación de teorías-, Markman destaca la siguiente cuestión: al aprender un lenguaje nativo, el niño es como ese extranjero que llega a un lugar desconocido en donde el idioma le es extraño y, en un intento por comunicarse, cuando un lugareño le señala en tal dirección y profiere «Gavagai» (señalando lo que podemos identificar como un conejo), ¿qué significado puede darle el niño a esa palabra? Siendo la primera vez que la escucha, ¿el niño atribuye su significado al conejo cuando está corriendo, o al conejo comiendo una zanahoria, al color del conejo o al conejo cuando está en el campo? De todos estos posibles significados -que el niño al igual que el extranjero- puede atribuirle a la palabra «Gavagai». ¿Cuál es el que el niño prefiere y por qué?

Los posibles significados dados a la palabra «Gavagai» -el conejo corriendo, el conejo comiendo una zanahoria, etc.pueden ser tomados como ejemplos de las ya mencionadas relaciones temáticas, que son importantes debido a que son las que el niño -y se dice que no sólo éste, sino también los adultos- encuentra más interesantes y más salientes en comparación con las relaciones taxonómicas. Debido a esto, en las teorías tradicionales de categorización, se cree que la categorización del niño se basa en las primeras, más bien que 
en las segundas, afirmación que Markman pretende desmentir (1989).

Por ello, y teniendo en cuenta que las relaciones temáticas son un modo de organización más accesible para los niños, Markman da evidencia de cómo este tipo de organización no es el único del que se sirve el niño, ya que «los niños pueden tener hipótesis implícitas sobre los posibles significados de las palabras que les ayudan a adquirir palabras para categorías. Incluso niños muy pequeños pueden ser conscientes del significado de una palabra» (1989: 27).

Siendo así, existe esta dualidad: el niño organiza el mundo por medio de relaciones temáticas y relaciones taxonómicas. A diferencia de lo que se creía -que el niño sólo clasificaba temáticamente-, Markman encontró que:

[...] en ausencia de etiquetas el niño frecuentemente organiza sobre la base de relaciones temáticas entre objetos, pero cuando un objeto es etiquetado, éste interpreta la etiqueta como refiriéndose a objetos que están relacionados taxonómicamente más que temáticamente. Por ejemplo, en ausencia de etiquetas, el niño selecciona un auto y un neumático como siendo el mismo tipo de cosa. Cuando el auto fue llamado 'un dax', sin embargo, y se le pidió al niño que encontrara otro 'dax', frecuentemente seleccionaba una bicicleta debido a que auto y bicicleta están en la misma categoría sueperordinaria, vehículos (1989: 219). 
Ellen Markman: Lenguaje y riqueza estructural vs categorías arbitrarias

Para Ellen Markman existe un continuo que, en un extremo, sitúa a las categorías arbitrarias y, en el otro, sitúa a las categorías naturales. Una de las características que hacen diferir a estos tipos de categorías es, principalmente, la riqueza de información que contienen, es decir, una categoría natural tiene una estructura interna ricamente correlacionada, mientras que con la categoría arbitraria ése no es el caso.

De esta diferencia fundamental derivan algunas otras, principalmente: 1) «las categorías ricamente estructuradas soportarán más inferencias que las categorías arbitrarias» y 2) «las categorías ricamente estructuradas serán vistas como capturando algo de importancia fundamental sobre un objeto, mientras que las categorías arbitrarias proveen información menos esencial» (1989: 226).

Los extremos de este continuo, según Markman, muestran una correlación «imperfecta pero útil» entre la riqueza de una categoría y la clase de forma gramatical, esto es, «los sustantivos tienden a apuntar a categorías de riqueza estructural, y los adjetivos tienden a señalar a categorías más arbitrarias» (1989: 227).

Integración de elementos

Ahora bien, dado que hemos expuesto de manera suficiente las ideas de Gentner y Markman, pretendemos integrar sus aportes de modo que nos permitan cumplir nuestro objetivo: caracterizar los predicados proyectables. La manera en la que integraremos los aportes de estas autoras es suponiendo que cada uno de sus resultados forman continuos -esto, por supuesto, tomando la idea de Gentner de usar la información como parte de un continuo-, y que estos continuos, a la 
vez, son las dimensiones de un espacio. Se pretende, entonces, que en esas dimensiones sea posible identificar palabras como verde y palabras como verzul, que son nuestro caso de estudio, y se refieren a predicados proyectables y no proyectables, respectivamente.

Para ser precisos con el uso de los términos, definimos dimensión como el «rango completo de variación» (Treisman, 1980: 99) o «el número de coordenadas necesarias para especificar la posición de un punto particular en el espacio» (Tanton, 2005: 136), o lo que es lo mismo, los posibles valores de variación que puede exhibir un fenómeno. Para dejar claro el uso de las dimensiones, especificaremos qué es un espacio descriptivo.

\section{Un espacio descriptivo}

En esta tesis, interpretaremos un espacio abstracto de descripción como un marco teórico que nos permite describir aspectos de un cierto fenómeno. Los espacios se construyen de manera análoga a las descripciones geométricas que son familiares en las matemáticas. En las ciencias naturales, los espacios abstractos se utilizan para describir aspectos cuantificables de los fenómenos. El ejemplo más simple de espacio abstracto es la descripción geométrica del espacio físico, al estilo de las descripciones utilizadas en la geometría analítica.

Ahora, para los propósitos de la tesis, una noción muy útil de espacio abstracto de descripción es la noción que interpreta a un espacio como una descripción que expresa los grados de libertad de un fenómeno, es decir, las formas en las que el fenómeno puede mostrar cambios. Esta forma de interpretar el espacio abstracto tiene la ventaja de que permite concebir las dimensiones como la descripción de las posibles 
variaciones de las propiedades involucradas en un fenómeno. Podemos decir que un espacio abstracto está compuesto de diferentes dimensiones. Recuérdese que el objetivo de esta tesis es utilizar los continuos de información propuestos por Gentner y Markman como base para modelar los fenómenos psicológicos que determinan la adquisición de palabras como verde y verzul. Atendiendo a este propósito, es claro que si la noción de espacio abstracto -tal como la hemos caracterizado arriba- cumple precisamente la finalidad de integrar dimensiones en un marco descriptivo, la noción de espacio es la herramienta idónea para cumplir el objetivo de ayudarnos a caracterizar un predicado proyectable.

Así, los continuos postulados por Gentner y Markman serán interpretados como las dimensiones constituyentes de un espacio informacional. Bajo esta interpretación, las dimensiones del espacio describen un aspecto de la información que el sujeto utiliza para adquirir palabras; y el espacio mismo, siguiendo con la misma interpretación, describe esa misma información de manera integral, es decir, no sólo un aspecto sino un fenómeno de manera más completa.

Para cumplir con la tarea de construir un espacio abstracto de descripción es preciso atender las siguientes condiciones.

Condiciones para la construcción del espacio

Para construir este espacio descriptivo es necesario mencionar algunas condiciones que muestren la relevancia de usar este modo de descripción de un fenómeno, es decir, que muestre que esa descripción será buena, simple y fiel con lo que ocurre en la realidad, y, al mismo tiempo, que nos permita ver la relevancia y pertinencia de los fenómenos psicológi- 
cos que explican Gentner y Markman para caracterizar predicados proyectables y no proyectables. Estas condiciones son tres: 1) la naturaleza del fenómeno, 2) la ortogonalidad, y 3) la organización de las dimensiones.

\section{Naturaleza del fenómeno}

Como ya se mencionó con anterioridad, los continuos que se usarán para interpretarlos como dimensiones de nuestro espacio, son los propuestos por Markman y Gentner. Por un lado, en el caso de Gentner, para el dominio cognitivo-dominio lingüístico se toma en cuenta la información que el niño tiene disponible como aprendiz del lenguaje, que es tanto la cognitivo-perceptual como la del lenguaje que escucha y pretende hablar. En estos dos flujos de información, en una primera etapa del desarrollo del niño, predomina el dominio cognitivo-perceptual, mientras que el dominio lingüístico forma parte de etapas posteriores de su desarrollo.

Por otro lado, en Markman, para la relación temáticataxonomía, se considera que también existe información de la que el niño puede servirse para aprender palabras y para agruparlas. La pregunta que se formula es:, ¿cómo es que un niño organiza la información cognitivo-perceptual o, incluso, la información obtenida del lenguaje mismo?

En el caso de categoría natural-categoría arbitraria, también de Markman, la separación que existe entre las diferentes clases de palabras, en las que se cree que unas -de corte natural- encierran una estructura correlacionada y, por tanto más rica, y que otras, -de corte arbitrario- son de estructura más bien empobrecida, contribuyen al aprendizaje de categorías por parte del niño.

Dicho lo anterior, hacemos evidente que este tipo de información nos da una pista más bien de tipo cognitivo que 
solamente de tipo pragmático sobre la preferencia de ciertas palabras sobre otras. Es decir, nos da razones para creer que el hecho de que en nuestro vocabulario existan palabras del tipo 'verde' no es necesariamente fortuito, apegado solamente al uso de esta palabra, si pensamos que nuestra otra opción -verzul- quedaría fuera de nuestro alcance en edades muy tempranas del desarrollo e incluso en etapas maduras. Así, pues, las propuestas de las autoras citadas anteriormente son relevantes para complementar la idea de que no es sólo la utilización de las palabras lo que hace que sean proyectables o no, sino más bien al flujo de información en el que se encuentre (dominio cognitivo-dominio lingüístico), su estructura interna y externa (relación temática-taxonomía) y su estructura interna (categoría natural-categoría arbitraria).

\section{Ortogonalidad}

Algo de suma importancia al construir nuestro espacio es reconocer cada una de nuestras dimensiones como independientes una de otra, es decir, que al unirlas en un espacio y localizar un punto en alguna de ellas no afecte la localización de éste en las dimensiones restantes. En palabras puntuales: ¿cómo sabemos que la dimensión de Gentner dominio cognitivo-dominio lingüístico es ortogonal ${ }^{7}$ a la dimensión de Markman categoría natural-categoría arbitraria, como primera instancia? O, lo que es lo mismo, ¿cómo sabemos que las propuestas de las autoras no se determinan de alguna manera?

Una de las respuestas más obvias es que se debe al tipo de fenómeno que quieren explicar. En el trabajo de Gentner

7 Entendiendo por ortogonal lo que, en términos, técnicos sería la intersección de dos líneas que forman un ángulo recto (Tanton, 2005: 369) y, por tanto, la independencia de la que se hablaba más arriba. 
sabemos que su interés se centra en las primeras palabras que el niño adquiere teniendo a su disposición dos corrientes de información: la cognitivo-perceptual y la del lenguaje que se habla (Gentner, 1982). Por otro lado, Markman se interesa por 1) cómo el niño organiza la información que, podemos decir (usando la terminología de Gentner), tiene a su disposición como corriente de información, y 2) saber cómo los niños aprenden categorías (y al proponer por qué la preferencia de unas sobre otras), basándose en lo que se considera categoría natural y categoría arbitraria.

Otra de las respuestas es que -aunque las autoras están tratando con un mismo tipo de fenómeno, que es el de la adquisición de palabras- podemos ver sus propuestas como complementarias más que como respuestas del mismo tipo. En el caso de las dimensiones antes citadas (dominio cognitivo-dominio lingüístico / categoría natural-categoría arbitraria), sabemos del primer binomio que, por un lado, tenemos dependencia total de la percepción y, por el otro, esta dependencia radica en el conocimiento del lenguaje adquirido durante el desarrollo. En el segundo binomio la diferencia de los conyuntos radica en la complejidad de las estructuras: la categoría natural está más ricamente estructurada y la adscripción a ésta es permanente, mientras que la categoría arbitraria tiene menos riqueza de información y la pertenencia a ella es más transitoria (Markman, 1989).

Como puede verse, dichas propuestas dirigen su atención a facetas distintas. En el caso del binomio de Markman, no se hace énfasis en que la percepción juegue un papel importante en la distinción de sus categorías. Sin embargo, en otro de sus trabajos menciona que las categorías de tipo natural «no son necesariamente identificadas por simples propiedades perceptuales» (Markman, 1986: 184), lo que lo 
distingue de manera explícita del conyunto «dominio cognitivo» del binomio dominio cognitivo-dominio lingüístico.

Cabe destacar que en el binomio relación temática-taxonomía, se hace alusión a un tipo de organización de la información, a saber, una organización por eventos o por clase, lo que lo diferencia del tipo de organización dictada por el binomio categoría natural-categoría arbitraria, donde ya se habla de clases (taxonomías) específicas. La independencia entre estos, pues, salta a la vista como obvia.

\section{Organización de las dimensiones}

Ahora bien, determinar la orientación de cada una de las dimensiones parece una tarea no improcedente; y es que, dependiendo de cómo fijemos cada dimensión, el espacio nos proporcionará una buena descripción y fidelidad con el fenómeno que representa.

Recordemos que el objetivo último de esta investigación es caracterizar un predicado proyectable, usando una representación formal que permita situar la región de los «verdes» y la región de los «verzules» ${ }^{8}$. Al identificar en nuestro espacio descriptivo la ubicación de verde y verzul, estaríamos mostrando una pauta para determinar la región de palabras proyectables y de palabras no proyectables, objetivo a largo plazo que se incluirá en futuras investigaciones. Así, pues, ¿cómo determinamos la organización de las dimensiones?

A pesar de que reconocemos la importancia de la organización del espacio descriptivo, la organización de las di-

8 Nos referimos a los «verdes» y a los «verzules» para hacer referencia a las "palabras como...», es decir, uno de los objetivos a largo plazo es que, al situar verde y verzul en el espacio, estaremos estableciendo qué palabras proyectables como verde se encuentran aledañas a éste o, lo que es lo mismo, determinar las regiones separadas, por supuesto, donde se sitúa la proyectabilidad y la no proyectabilidad. 
mensiones es una propuesta provisional, es decir, que está sujeta a mejoras. Esto por la única razón de que no tenemos de cierto si para cada uno de los continuos exista un origen. Por ejemplo, en el caso del binomio dominio cognitivo-dominio lingüístico, el origen está claramente ubicado en el dominio cognitivo, es decir, el fenómeno que Gentner explica marca su inicio en el dominio cognitivo. Lamentablemente, esto no está determinado para los otros continuos. Lo que sí determinamos, a modo de convención, es que las dimensiones tuvieran un origen común, que cada una de ellas fueran ortogonales y que siguieran indefinidamente.

Función del espacio descriptivo para caracterizar un predicado proyectable

Dada la propuesta que hemos esbozado en apartados anteriores, cabe preguntarse si efectivamente es posible representar a verde y verzul -ejemplos tomados de Goodman y de nuestro estudio de caso- en el espacio descriptivo. Si es posible representar $\mathrm{o}$, si se prefiere, situar a verde y verzul en cada una de las dimensiones; lo siguiente es saber si verde y verzul se localizarán en áreas distintas del espacio descriptivo, lo cual permitirá separarlos el uno del otro.

Para dar respuesta a lo anterior, tomaremos cada uno de los continuos -que serán interpretados como dimensiones- y en ellos localizaremos el punto distintivo de verde y el punto distintivo de verzul. 
Dominio cognitivo-dominio lingüístico

Comencemos con Gentner -trabajo del que surgió esta ideay su binomio dominio cognitivo-dominio lingüístico. Proponemos que la localización ${ }^{9}$ de verde y verzul sea la que se muestra a continuación en la figura 1.

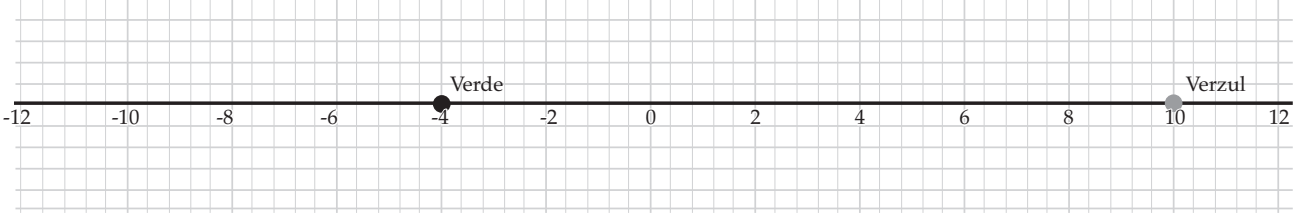

Figura 1. Dominio cognitivo representado de lado izquierdo y dominio lingüístico de lado derecho.

Según la definición de Gentner, en el dominio cognitivo encontramos «conceptos que surgen de una esfera cognitivoperceptual y simplemente son nombrados por el lenguaje» (individuados sobre una base perceptual); mientras que el dominio lingüístico se caracteriza por que «el mundo presenta pedazos cuyo agrupamiento no está pre-ordenado, y el lenguaje dice cómo esos pedazos se combinan en conceptos» (2001: 215).

Atendiendo a las definiciones, creemos que verde podría situarse en el dominio cognitivo, dado que éste es detectable simplemente al contacto con el entorno; nos viene dado por la experiencia sensorial. En el caso de verzul, su localización cae al otro extremo del continuo, es decir, en el dominio lingüístico, dado que, en principio, su definición es una definición aprendida, es decir, alguien -en este caso,

9 Cabe señalar que la localización de cada uno de los puntos no es una localización concluyente; lo presento como hipótesis y, por supuesto, está sujeta a discusión. 
Goodman- nos tiene que instruir respecto a la aplicación de tal predicado, haciendo uso de herramientas del lenguaje como la lógica. Luego, dentro de su definición -verzul «se aplica a todas las cosas examinadas antes de $t$ sólo en caso de que sean verdes, pero a otras cosas sólo en caso de que sean azules» (Goodman; 1983: 74) -existe una condición lógica que no debe de ignorarse, a saber, «si algo es verde y es examinado antes de $t$, entonces es verzul» $\mathrm{y}$ «si algo es azul y no es examinado antes de $t$, entonces es verzul» ${ }^{10}$.

Lo que esto implica es que, para que un niño pudiera aplicar correctamente el predicado verzul tendría que tener conocimiento de conjunciones, por ejemplo, que entran en el dominio lingüístico, así como de todo lo que antecede a ese conocimiento según el continuo de Gentner.

Así pues, estas son las razones por las que creemos que, en el continuo propuesto por Gentner, verde y verzul ocuparían esas locaciones.

Relaciones temáticas-taxonomía

Contrario a lo que ocurre con el trabajo de Gentner, en cuyo caso podemos argumentar la localización de verde y verzul más fácilmente, en el caso de la Adquisición de términos de categoría de Ellen Markman, el asunto es menos sencillo; sin embargo, baste recordar que la localización de los puntos es apenas una hipótesis que estamos proponiendo y queda sujeta a mejoras.

En un principio, proponemos que verde quede situado en el extremo perteneciente a las relaciones temáticas y

10 Esto atendiendo al análisis estrictamente lógico del término: donde se tiene una condición (C1) que dicta: O se observa antes de $t$ y O es verde. Si C1 se cumple, entonces «verzul» es aplicable a O. Si C1 no se cumple, entonces «verzul» es aplicable a O si y sólo si O es azul. 
verzul en el extremo contrario, en el de taxonomía, como se muestra a continuación en la figura 2.

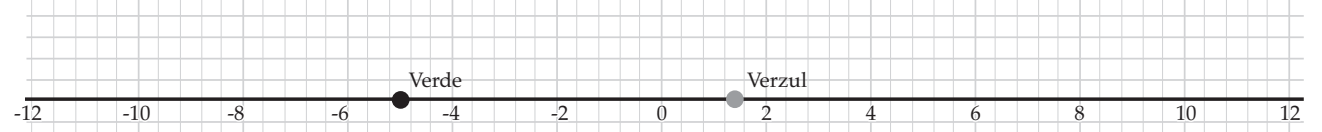

Figura 2. Relaciones temáticas representado de lado izquierdo y taxonomía de lado derecho.

Las razones que nos llevan a justificar esta decisión son que, por un lado, verde puede ser visto como una relación existente entre objetos: entre los que comparten el color verde. Es decir, si bien verde puede ser visto como un modo de organizar objetos, esa organización se llevaría a cabo en virtud de la propiedad «ser verde»: un niño podría categorizar el follaje de un árbol, un limón y una pelota, en virtud de que los tres comparten la propiedad «verde». Considérese también que las relaciones temáticas resultan más interesantes para los niños, o les resultan más simples (Markman, 1989), como pasaría en el caso de una palabra de color como verde que forma parte de los «colores focales», según las clasificaciones hechas en investigaciones de términos de color, por ser, quizá, más saliente que otros colores como el gris (Taylor, 1995; Pitchford, 2002).

Algo que resulta atractivo de las diferencias que enuncia Markman entre relaciones temáticas y taxonomía, es que las relaciones temáticas no pueden fungir ellas solas como bases para la formación de categorías (Markman, 1989), cosa que con la categorización taxonómica sí sucede. Y es que hay que recordar que la categorización taxonómica es como un listado de características funcionales, perceptuales o algo 
más abstracto, necesarias y suficientes que deben tener -ya sea absoluta o probabilísticamente-cada uno de los miembros de determinada categoría. En el caso de verde, si se piensa, no hay mucho que enlistar, sólo una perogrullada como: que los objetos que entren dentro de la categoría deben ser verdes. Verzul, por otro lado, enlista al menos dos características necesarias y suficientes que debe tener aquello que quepa en esta categoría, a saber: a) ser observado antes de un tiempo $t$ y ser verde, y b) no ser observado antes de un tiempo $t$ y ser azul.

Si bien lo anterior no muestra de manera muy obvia la posible diferencia entre verde y verzul, nos permite considerar lo siguiente: que al ser las relaciones temáticas un análisis externo de los objetos para organizarlos en una categoría se adecúa, más o menos bien, a un tipo de organización como verde, que considera como característica única para pertenecer a dicha categoría «ser verde». Por su parte, la categorización taxonómica, al ser, por el contrario, un análisis interno de una categoría, busca características menos evidentes que las que se dan «a primera vista»; es por ello que verzul sería parte de la taxonomía en el continuo.

Nótese que nuestra justificación de localización en este continuo es menos obvia que la que hemos propuesto en el continuo de Gentner, como mencionamos más arriba. No obstante, esperamos que estos indicios sean suficientes para transmitir la intuición que nos llevó a la decisión de localizar a verde y verzul de ese modo.

Categoría natural-categoría arbitraria

Como última dimensión tenemos la categoría natural-categoría arbitraria de Markman. La localización de verde y verzul se muestra a continuación en la figura 3: 


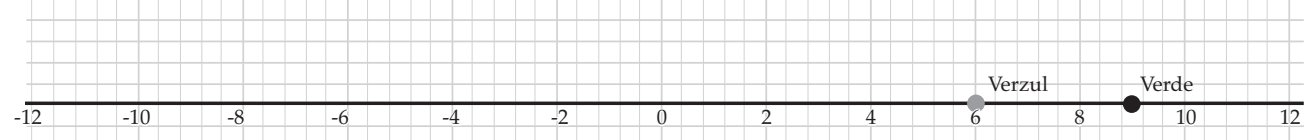

Figura3.Categoríanaturalrepresentadadeladoizquierdoycategoríaarbitrariadeladoderecho.

Para Markman estos tipos de categorías se diferencian por la riqueza de información correlacionada dentro de una categoría y otra. De una categoría natural, podemos esperar más inferencias que de una categoría arbitraria, por ejemplo: «pájaro», como categoría natural, arroja más información que «cosa blanca», a saber: tener alas, poner huevos, volar, etc. La categoría arbitraria funge como apoyo al robustecimiento de las categorías naturales; la primera contiene términos que refieren, mientras que la segunda contiene términos que describen. Siendo esto así, Markman identifica la categoría arbitraria con los adjetivos y la categoría natural con los sustantivos.

Dicho lo anterior, no puede ignorarse que verde y verzul no encierran en sí mismos una riqueza estructural al modo propuesto por Markman, es decir, no contienen grandes racimos de información y, por tanto, no soportan inferencias. Cumplen, básicamente, el papel de adjetivos, no obstante, verzul sería un caso especial de adjetivo. Para sustentar esta afirmación me apoyaré en el trabajo de Susan Gelman y John Coley, con el fin de que complementen la idea que retomamos de Markman.

Según Gelman y Coley (2008), existen propiedades que transmiten la naturaleza psicológica de la clase natural, es decir, aquello que hace que la clase natural sea tomada tal cual por el agente. Son seis propiedades que, digámoslo así, deben cumplirse para llamar a una categoría natural a saber: potencial de riqueza inductiva, bases no obvias, esencia, 
existencia de anomalías, división de la labor lingüística y corregibilidad. Según los autores, habiendo identificado el tipo de categoría que tenemos, se tendrá el impacto en las hipótesis, es decir, saber si dichas categorías se pueden usar para generar hipótesis e inferencias inductivas (Gelman, 2008).

Si bien en El nuevo enigma, Goodman nos dejó claro que verzul no es un término con el cual se puedan hacer proyecciones, debido al condicional implicado en su aplicación, verzul parece acatar al menos una de las propiedades que mencionan Gelman y Coley, y si bien esto no lo haría acreedor del calificativo «categoría natural» sí lo separa de verde de una manera significativa, haciendo que verzul no sea un adjetivo común.

La propiedad que consideramos que verzul cumple es la de «bases no obvias»; a lo que los autores se refieren con bases no obvias es a que las categorías de tipo natural «capturan semejanzas profundas que no siempre son inmediatamente obvias» (Gelman, 2008: 151); y es que, analizando verzul, sabemos que tiene semejanzas con otro tipo de términos que no son inmediatamente dadas, es decir, con frases como «presidente de la república», «rector de la universidad», «jefe de grupo», etc. El tipo de semejanza entre estos términos es el condicional implicado para su aplicación, es decir, verzul tiene cierto referente hasta determinado tiempo al igual que, por ejemplo, presidente de la república. Además, si bien estos términos son términos que refieren -usando el calificativo de Markman al que se refiere con los adjetivos-, contienen una cantidad de información más basta, comparada con un adjetivo como verde.

Ya que hemos localizado en cada uno de los continuos los puntos distintivos de verde y verzul, queda incorporarlos como dimensiones en el espacio descriptivo. 


\section{Construcción del espacio}

Tomando valores provisionales en cada una de las dimensiones, tenemos una serie de coordenadas que nos permiten situar al punto que denominamos verde y al punto que denominamos verzul. Nuestro espacio descriptivo queda representado a continuación, en la figura 4 :

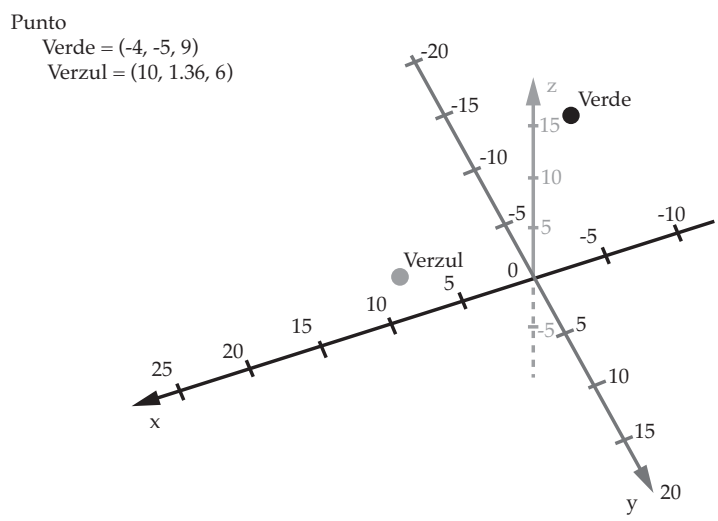

Figura 4. En el eje $x$ se representa la dimensión dominio cognitivo-dominio lingüístico; en el eje y la dimensión relación temática-taxonomía y, en el eje $z$, categoría natural-categoría arbitraria.

Nuestro espacio hace lo que esperábamos: caracteriza y diferencia verde de verzul, es decir, un predicado proyectable de un predicado no proyectable, permitiéndonos distinguir claramente uno de otro.

Al obtener un espacio con regiones específicas para palabras como verde y palabras como verzul, ampliamos el margen descriptivo de lo que es un predicado proyectable y uno no proyectable. Consideramos que verde y verzul tienen diferencias en tanto su adquisición, las cuales podemos enunciar como sigue: 
Verde

1. Palabras como verde son adquiridas de manera perceptual, es decir, lo perceptual es lo único necesario para identificar o señalar dicha palabra.

2. La palabra verde es más accesible y saliente para un agente-en este caso, un niño-comparada con verzul. Esta característica es un cupla entre el punto 1 y la explicación dada por Goodman al responder sobre la preferencia de unas palabras sobre otras (el uso de las palabras), ya que creemos que al dar ambas palabras a un niño para que agrupe objetos bajo éstas, nuestra intuición es que la reacción hacia verde será más rápida que la reacción hacia verzul, es decir, el niño tomará las características perceptuales de los objetos a favor de verde, dejando lo que no-es-verde en la categoría de verzul; esto dado que a) verde le resulta más familiar que verzul, b) verde es un término de color (que el niño sabe que es un término de color- que comprende y produce a temprana edad -de nuevo, pensando en las investigaciones sobre la adquisición de términos de color-.

3. Palabras como verde describen características de los objetos que son transitorias e incluso prescindibles; que no hacen más que modificaciones a las categorías instauradas como tales. Así, verde funge como apoyo a la construcción de categorías naturales ${ }^{11}$.

11 Esto nos hace pensar que palabras como verde no podrían estar incluidas en cualquier generalización, o quizás ser información irrelevante o de poco interés científico para considerarla como proyectable. Pensando en Gelman y Coley (2008), mientras que «las categorías artificial o arbitrarias nombran para expresar cierta cualidad» $\mathrm{y}$ «no cambian con la adición de nueva información», las categorías naturales «ayudan a capturar, lo mejor posible, la verdadera estructura del mundo» (156-159); y desde un inicio esto iría en contra de lo propuesto por Goodman. 
Verzul

1. Palabras como verzul requieren del lenguaje como herramienta para poder ser comprendida, es decir, además de lo perceptual es necesaria la instrucción del lenguaje y cierta disposición cognitiva para poder comprender y producir dicha palabra.

2. Palabras como verzul se aplican a cuestiones de más alto nivel, es decir, como se mencionó anteriormente, no sólo a cuestiones perceptuales sino a conocimientos del lenguaje más sofisticados como el uso de condicionales lógicos y temporales.

Con la caracterización anterior, hemos logrado alcanzar el objetivo principal de este texto: identificar los rasgos distintivos de verde y verzul. Sin embargo, esto apenas es el primer paso para una tarea más grande y ambiciosa: la generalización de nuestros supuestos, es decir, que las características que hemos reunido con estos casos de estudio en particular, sean aplicables para la región completa -pensando en el espacio descriptivo- de palabras proyectables y palabras no proyectables.

\section{Conclusiones}

El objetivo principal de esta investigación fue la caracterización de un predicado proyectable; para ello, propusimos usar las teorías y resultados en adquisición de palabras, particularmente, de los trabajos de Dedre Gentner y Ellen Markman. Haciendo uso de esto propusimos un método que nos permitiera alcanzar la caracterización de los predicados proyectables - particularmente de verde- en contraposición 
a predicados no proyectables como verzul; el método que propusimos fue un espacio descriptivo. Consideramos al espacio descriptivo como una de las maneras más simples y fieles para representar el fenómeno que nos atañe: los predicados proyectables.

A lo largo de la investigación, fuimos construyendo el argumento que nos permitiera sustentar la insuficiencia de la respuesta proporcionada por Nelson Goodman a su idea de que es el uso y el atrincheramiento lo que determina que palabras como verde sean proyectables. En principio, mostramos un panorama general del concepto de la inducción, así como de las ideas centrales que aparecen en El nuevo enigma de la inducción donde aparece por primera vez la propuesta de predicado proyectable. Posteriormente, pensando en lo parco de la propuesta de Goodman y en querer dar una alternativa con bases empíricas sobre los predicados proyectables, formulamos nuestra pregunta de investigación: ¿pueden las teorías y resultados en adquisición de palabras -particularmente, los trabajos de Dedre Gentner y Ellen Markman- ofrecer una caracterización que nos permita diferenciar predicados proyectables de predicados no proyectables? Si esto es así, ¿cómo es que lo logran?

Luego, nos adentramos en las investigaciones en adquisición de palabras con Gentner y Markman, reseñando las partes de sus trabajos que nos parecían útiles para nuestros propósitos. Después, propusimos integrar estas propuestas en un todo como dimensiones que conformaron el espacio descriptivo.

El espacio descriptivo que propusimos como la forma teórica de describir un fenómeno, cumple, no obstante, tres condiciones que permiten que nuestra descripción sea una buena descripción, simple y fiel al fenómeno de los predica- 
dos proyectables: la naturaleza del fenómeno -es decir, de cada una de las propuestas de Gentner y Markman-, la ortogonalidad -la independencia de cada una de las dimensiones-, y la organización de las dimensiones.

Finalmente, mostramos cómo es que funcionan cada una de las dimensiones situando verde y verzul -los puntos identificados en las dimensiones-, que fueron nuestro caso de estudio. Integradas las dimensiones en el espacio descriptivo, pudimos corroborar que verde y verzul se sitúan en sitios distintos del espacio, lo que nos permitió alcanzar nuestro objetivo: caracterizar un predicado proyectable $y$, en consecuencia, uno no proyectable. Es decir, y atendiendo a la definición de «caracterizar», determinamos los rasgos distintivos de verde y verzul, lo que los diferencia a uno del otro expresados en un espacio físico.

Con el objetivo alcanzado, queda pendiente, eventualmente, el uso de una métrica en el espacio, que nos permitiría separar un predicado proyectable de un predicado no proyectable, haciendo separables por un «muro» las regiones del espacio pertenecientes a palabras proyectables y las regiones pertenecientes a las no proyectables, permitiendo identificar los gradientes para palabras pertenecientes a una región o a otra, es decir, saber cuáles son más proyectables y cuáles menos proyectables.

\section{Bibliografía}

Audi, R. (1999). The Cambridge Dictionary of Philosophy. Estados Unidos de América: Cambridge University Press.

Gelman, S., y Coley, J. (2008). Language and Categorization: The Acquisition of Natural Kind Terms. En Gelman, S. 
y Byrnes J. (Eds.) Perspectives on Language and Thought. Estados Unidos de América: Cambridge University Press.

Gentner, D. y Boroditsky, L. (2001). Individuation, relativity and early word learning. En Bowerman, M., y Levinson, S. (Eds.) Language acquisition and conceptual development. Reino Unido: Cambridge University Press.

Gentner, D. (1982). Why Nouns are Learned Before Verbs: Linguistic Relativity versus Natural Partitioning. En Kuczaj II, S. (Ed.) Language Development, Volume 2: Language, Thought and Culture. Estados Unidos de América: Lawrence Erlbaum.

Goodman, N. (1953). Fact, Fiction and Forecast. Estados Unidos de América: Harvard University Press.

Griffiths, T. y Tenenbaum, J. (2009). Theory-Based Causal Induction. Psychological Review. (4). 661-716.

Markman, E. y Gelman, S. (1986). Categories and Induction in Young Children. Cognition. (23). 183-2009.

Markman, E. (1989). Categorization and Naming in Children: Problems of induction. Estados Unidos de América: MIT Press.

Pitchford, N. y Mullen, K. (2002). Is The Acquisition of BasicColour Terms in Young Children Constrained? Perception. (31). 1349-1370.

Rheinwald, R. (1993). An Epistemic Solution to Goodman's

New Riddle of Induction. Synthese. (95). 55-76.

Tanton, J. (2005). Encyclopedia of Mathematics. Estados Unidos de América: Facts On File, Inc.

Treisman, A. y Gelade, G. (1980). A Feature-Integration Theory of Attention. Cognitive Psychology. (12). 97-136. 


\section{Resumen}

La presente investigación tiene como objetivo principal caracterizar los predicados proyectables haciendo uso de las teorías y resultados encontrados en las ciencias cognitivas, particularmente, de las investigaciones en adquisición de palabras. Para identificar los rasgos característicos de un predicado proyectable y un predicado no proyectable, sugerimos la creación de un espacio descriptivo que nos permita representar y separar un predicado que es usado en nuestras inducciones - predicado proyectable- de otro que no puede ser usado en ellas -predicado no proyectable-. La idea de reunir el trabajo teórico de la filosofía y el trabajo empírico de la adquisición de palabras, permite tanto naturalizar los aportes del filósofo Nelson Goodman, como estimular el trabajo transdisciplinar.

Palabras clave: ciencias cognitivas, espacio descriptivo, inducción, predicado proyectable, verde, verzul.

\section{Abstract}

The main objective of this research is to characterize the projectible predicates using the theories and the results in the cognitive sciences, particularly, in the research about acquisition of words. In order to identify the characteristics of a projectible predicate and of a non-projectible predicate, we suggest the creation of a descriptive space that allows us to represent and separate a predicate that is used in our inductions - projectible predicate-from another that can't be used in them - non-projectible predicate-. The idea of bringing together the theoretical work of philosophy and the empirical work of words acquisition, naturalizes the contributions 
of the philosopher Nelson Goodman, as well as stimulate transdisciplinary work.

Key words: cognitive sciences, descriptive space, induction, projected predicate, green, grue. 